\title{
Stage IIIA Small Intestinal, Esophageal, Colorectal, Mesenteric, and Peritoneal Gastrointestinal Stromal Tumor AJCC v8
}

National Cancer Institute

\section{Source}

National Cancer Institute. Stage IIIA Small Intestinal, Esophageal, Colorectal, Mesenteric, and Peritoneal Gastrointestinal Stromal Tumor AJCC v8. NCI Thesaurus. Code C136784.

Stage IIIA includes: (T1, N0, M0, High Mitotic Rate); (T4, N0, M0, Low Mitotic Rate). T1: Tumor measuring $2 \mathrm{~cm}$ or less. T4: Tumor measuring more than $10 \mathrm{~cm}$ in greatest dimension. NO: No regional lymph node metastasis or unknown lymph node status. M0: No distant metastasis. High Mitotic Rate: Over 5 mitoses per 5 square millimeters, or per 50 HPF. Low Mitotic Rate: Five or fewer mitoses per 5 square millimeters, or per 50 HPF. (AJCC 8th ed.) 